\title{
analyse des phénomènes vibratoires dûs aux tirs en tunnels
}

\author{
par \\ P. Allard \\ Laboratoire Régional des Ponts et Chaussées, Aix-en-Provence \\ A. Rozière \\ S.N.C.F.-Ouvrages d'Art, Paris
}

RESUME : Des tirs de mines sont exécutés dans des tunnels SNCF pour des travaux de reprise en sous-oeuvre : abaissement de plate-forme, approfondissement ou créations de niches supplémentaires en piedroits.

Contrairement aux contrôles classiques, sur des constructions indépendantes du site miné et situées à des distances d'une dizaine à quelques centaines de mètres des tirs, les mesures de contrôle en tunnels sont réalisées en plaçant les capteurs à seulement quelques mètres du tir. Dans ce cas, la vibration présente un spectre de fréquence très large avec des niveaux les plus élevés entre 200 et $1000 \mathrm{~Hz}$.

L'étude présentée comporte l'analyse des vibrations détectées en différents points d'une section de tunnel, pendant des tirs en niche ou des tirs en plate-forme. L'analyse porte sur l'ensemble des impulsions, d'une volée à micro-retards et sur les 2 ou 3 premières impulsions de la même volée. Notre étude porte sur les spectres de fréquences mais également sur l'évolution du signal temps en fonction de la gamme de fréquences, avec un système de filtrage passe-bas.

En conclusion, nous proposons quelques principes méthodologiques pour l'étude des risques encourus sur l'ouvrage au cours du chantier de minage.

\section{INTRODUCTION}

Les mesures et contrôles réalisés dans les tunnels SNCF, au cours de chantiers de minage ont pour but de s'assurer que l'énergie dégagée par l'explosif et dissipée sous la forme de vibration, est réduite au niveau le plus faible possible pour ne pas provoquer de dégats sur l'ouvrage.

Corrélativement cette énergie de 1'explosion sera utilisée au mieux pour fragmenter et abattre la roche.

Les sollicitations dynamiques répétées pourraient, en effet, modifier la résistance naturelle du massif entrainant une nouvelle sollicitation du revêtement.

L'exposé qui suit présente des résultats d'enregistrements au cours de tirs de mines réalisés dans des tunnels de la ligne SNCF NARBONNE - PORT-BOU pour la mise au gabarit électrique.

La formation géologique est constituée par des schistes métamorphiques anciens.

\section{TECHNOLOGIE ET METHODE D'ETUDE}

Les mesures de vibrations sont réalisées avec des ensembles tridirectionnels de géophones d'un modèle courant qui présententune fréquence de résonance de $4,5 \mathrm{~Hz}$.

Les problèmes de bande passante peuvent se poser principalement vers les hautes fréquences.

Quelques étalonnages réalisés sur un pot vibrant entre $5 \mathrm{~Hz}$ et $1500 \mathrm{~Hz}$, montrent que au-dessus de $300 \mathrm{~Hz}$ les problèmes se posent principalement au niveau des fixations des capteurs, avec des pics de résonances sur des bandes de fréquences très étroites entre 750 et $800 \mathrm{~Hz}$ et vers $1500 \mathrm{~Hz}$.

Des systèmes de fixation avec des bridages rigides nous ont permis de vérifier que la réponse intrinsèque de certains capteurs est constante à 5 \% près jusqu'à plus de $1000 \mathrm{~Hz}$. 
L'enregistrement des vibrations est réalisé en analogique sur bande magnétique en modulation de fréquence avec une bande passante de 0 à $1250 \mathrm{~Hz}$ et une dynamique de $52 \mathrm{~dB}$.

L'exploitation est réalisée :

- d'une part sur le signal en fonction du temps de la forme $y=f(t)$. Ce type d'enregistrement nous permet de visualiser les signaux émis par chaque amorce de tir. Il peut s'agir de micro-retards à $25 \mathrm{~ms}$ ou de retards à $500 \mathrm{~ms}$;

- d'autre part sur un spectre de fréquences avec une représentation de la forme $\mathrm{Y}=\mathrm{F}(\mathrm{N})$ Cette fonction de la fréquence est obtenue par le calcul de la transformée de Fourier du signal-temps.

L'analyseur utilisé (fig. 1) échantillonne le

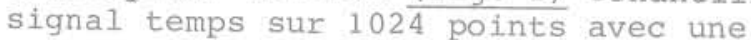
fréquence égale à 2,56 fois la fréquence maximale d'analyse.

L'analyse est alors réalisée sur 400 canaux

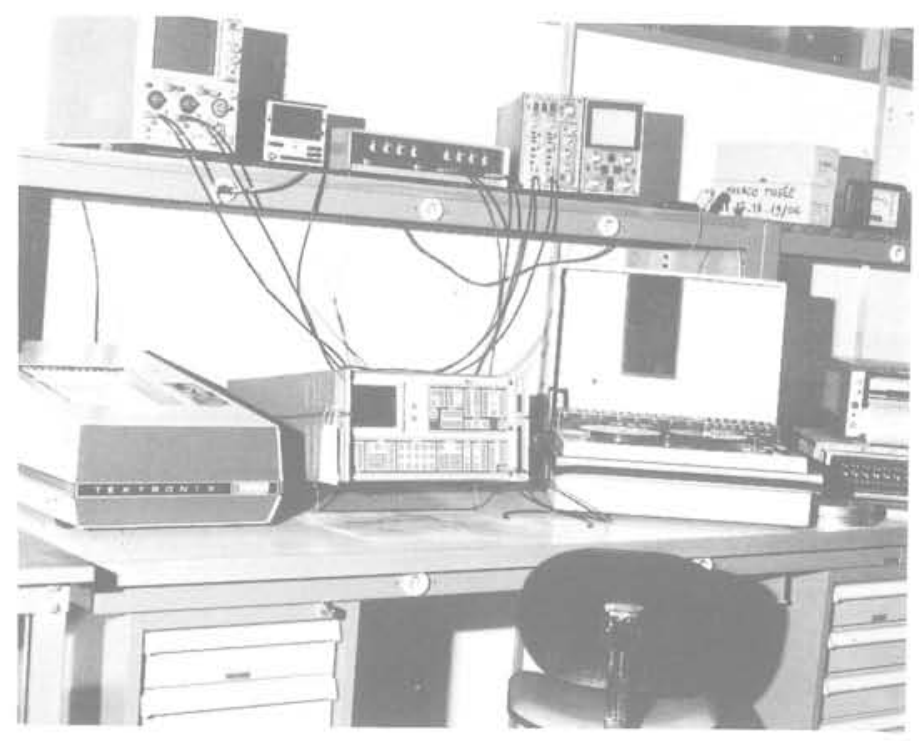

Fig. I CHAINE D'ANALYSE DES VIBRATIONS

Nous utiliserons une analyse sur l'ensemble des impulsions pendant une durée de $400 \mathrm{~ms}$. Nous obtiendrons alors 400 canaux d'analyse sur la bande $0-1000 \mathrm{~Hz}$.

Pour réaliser l'analyse sur un temps plus réduit, permettant l'échantillonage des 2 ou 3 premières impulsions seulement (deux ou trois micro-retards) nous réaliserons un échantillonnage sur $40 \mathrm{~ms}$ ou $80 \mathrm{~ms}$.

L'analyse sera alors présentée sur la bande $0-10 \mathrm{kHz}$ ou $0-5 \mathrm{kHz}$. Le signal sera contenu seulement sur 100 bandes d'analyse entre 0 et $2500 \mathrm{~Hz}$ ou entre 0 et $1250 \mathrm{~Hz}$.

Afin de comparer différents spectres et de calculer des fonctions et tranferts nous réaliserons des rapports de spectres.
Le filtrage, en passe-bas des signaux reproduit grace à l'enregistreur magnétique nous permettra de connaitre la forme des impulsions en fonction de la bande passante.

En raison de la nature impulsionnelle des signaux, nous utiliserons un filtre, tel que le temps de propagation de groupe dans la bande passante soit optimisé. Un tel filtrage sera décrit par la légende suivante :

F.D - (flat delay) P.B - (passe bas) X Hz

Pour les fréquences supérieures à X l'atténuation est de $48 \mathrm{~dB} /$ octave ou $96 \mathrm{~dB} /$ octave. $A$ la fréquence X 1 'atténuation est de $16 \mathrm{~dB}$. $\mathrm{L}$ 'atténuation à $3 \mathrm{~dB}$ est à considérer à la fréquence $\mathrm{x} / 2 \mathrm{~Hz}$.

L'étude que nous proposons, comporte :

L'analyse en amplitude et en fréquence de signaux dêtectés en différents points d'une section de tunnel lors de tirs dans des niches à créer, ou à approfondir et des tirs en pied-droit.

Nos observations porterons sur les spectres de fréquences, et les amplitudes en fonction de la bande passante avec des filtrages en passe bas.

\section{IMPLANTATION DES CAPTEURS}

Nous prendrons en considération 3 capteurs disposés comme indiqué sur la fig. l en section transversale du tunnel.

Le capteur $C$ est situé latéralement à $2,50 \mathrm{~m}$ du bord de la niche minée et à $1,50 \mathrm{~m}$ au-dessus des voies.

Les capteurs D et G sont situés dans le même plan de section transversale que le capteur C. Sur tous ces capteurs les 3 directions seront repérées de la façon suivante :

H1 : direction horizontale parallèle à l'axe du tunnel

H2 : direction horizontale perpendiculaire à l'axe du tunnel

$\mathrm{V}$ : direction verticale

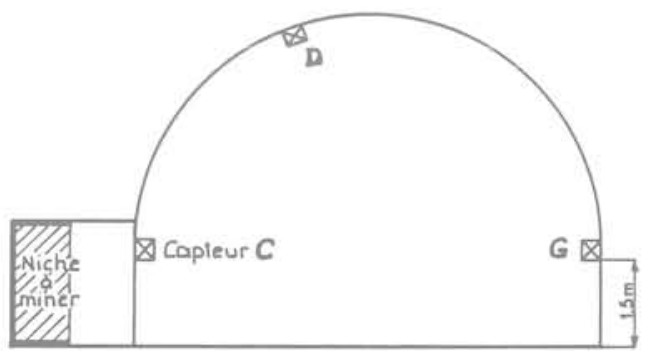

Fig. 2 POSITIONNENENT DE 3 CAPTEURS SUR UNE
SECTION POUR UN TIR DE NTCHE 

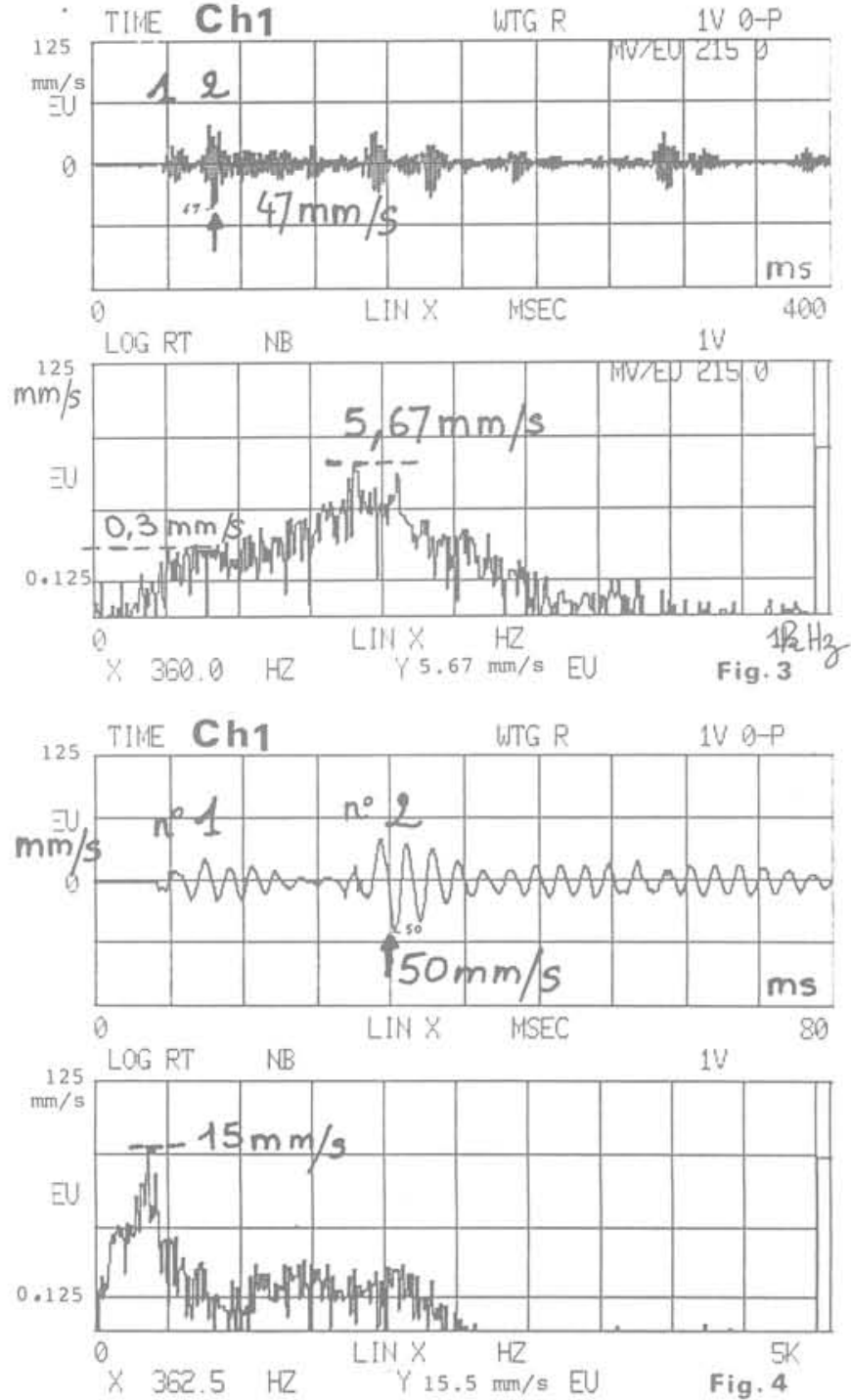

Fig 3 : Signal détecté sur le capteur $\mathrm{Ch}_{1}$ lors d'un tir de bouchon en approfondissement de niche.

Ce tir est constitué par 15 forages en $38 \mathrm{~mm}$ de diamètre suivant une maille de $0,30 \times 0,30 \mathrm{~m}$ la charge par forage est de $100 \mathrm{~g}$. Le signal temps nous permet d'observer sur $400 \mathrm{~ms}$ les impulsions consécutives aux 13 numéros de micro-retards. Le niveau maximal est obtenu sur le micro-retard $\mathrm{n}^{\circ} 2$ avec $47 \mathrm{~mm} / \mathrm{s}$.

Le spectre met en évidence une amplitude maximale sur le canal $360 \mathrm{~Hz}$ avec un niveau de $5,67 \mathrm{~mm} / \mathrm{s}$ zéro-crête.

Notons un palier sur le spectre entre 100 et $200 \mathrm{~Hz}$, mais à des niveaux d'environ $0,3 \mathrm{~mm} / \mathrm{s}$.

La fig. 4 présente le signal consécutif au premier, second et troisième numéro d'amorce du tir décrit ci-dessus.
Sur la plage de fréquences 0-1000 Hz le spectre est semblable à celui de la fig. 3 . Le niveau maximal est de $15,5 \mathrm{~mm} / \mathrm{s}$ sur le canal 362,5 Hz (largeur du canal : 12,5 Hz).

Des analyses du même type réalisées sur les autres capteurs et sur les 3 directions mettent en évidence un caractère identique des spectres, quelque soit le nombre des impulsions.

La plage de fréquences des signaux délivrés par le capteur Chl avec une dynamique de $30 \mathrm{c} B$ est comprise entre $100 \mathrm{~Hz}$ et $600 \mathrm{~Hz}$.

Effectuons une analyse du même type sur le signal délivré par le géophone vertical du capteur D, au cours du même tir. (fig. 5 et 6 )
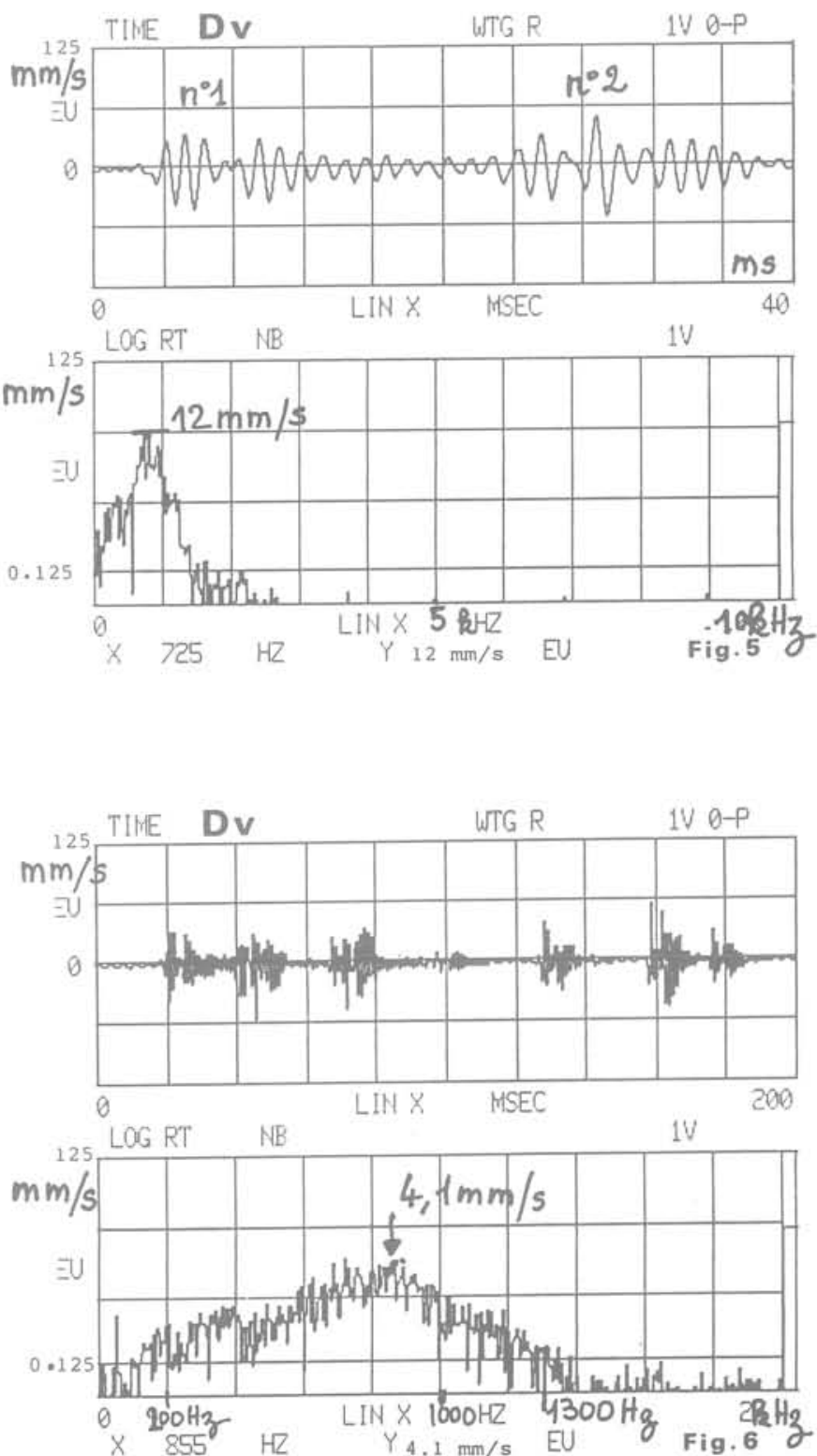
Cette analyse confirme les propos précédents concernant la plage de fréquences en fonction du nombre d'impulsions.

La fig. 5 présente l'analyse des 2 impulsions relatives aux micro-retards $n^{\circ} 1$ et 2 .

La bande de fréquences totale des signaux tant sur la fig. 5 que sur la fig. 6 est plus large que celle observée sur le capteur C (fig, 4).

La valeur maximale du spectre est obtenue sur une plage de fréquences de $725 \mathrm{~Hz}$ à $855 \mathrm{~Hz}$.

Avec une dynamique de $30 \mathrm{~dB}$ sous le niveau maximal du spectre on trouve une plage de fréquences de $100 \mathrm{~Hz}$ à $1300 \mathrm{~Hz}$. Cette dernière fréquence correspond d'ailleurs à la coupure hautes fréquences de l'enregistrement magnétique.

Des enregistrements analogues au cours de tirs en plate-formes ou de tirs de longrines présentent également une plage de fréquences très large.
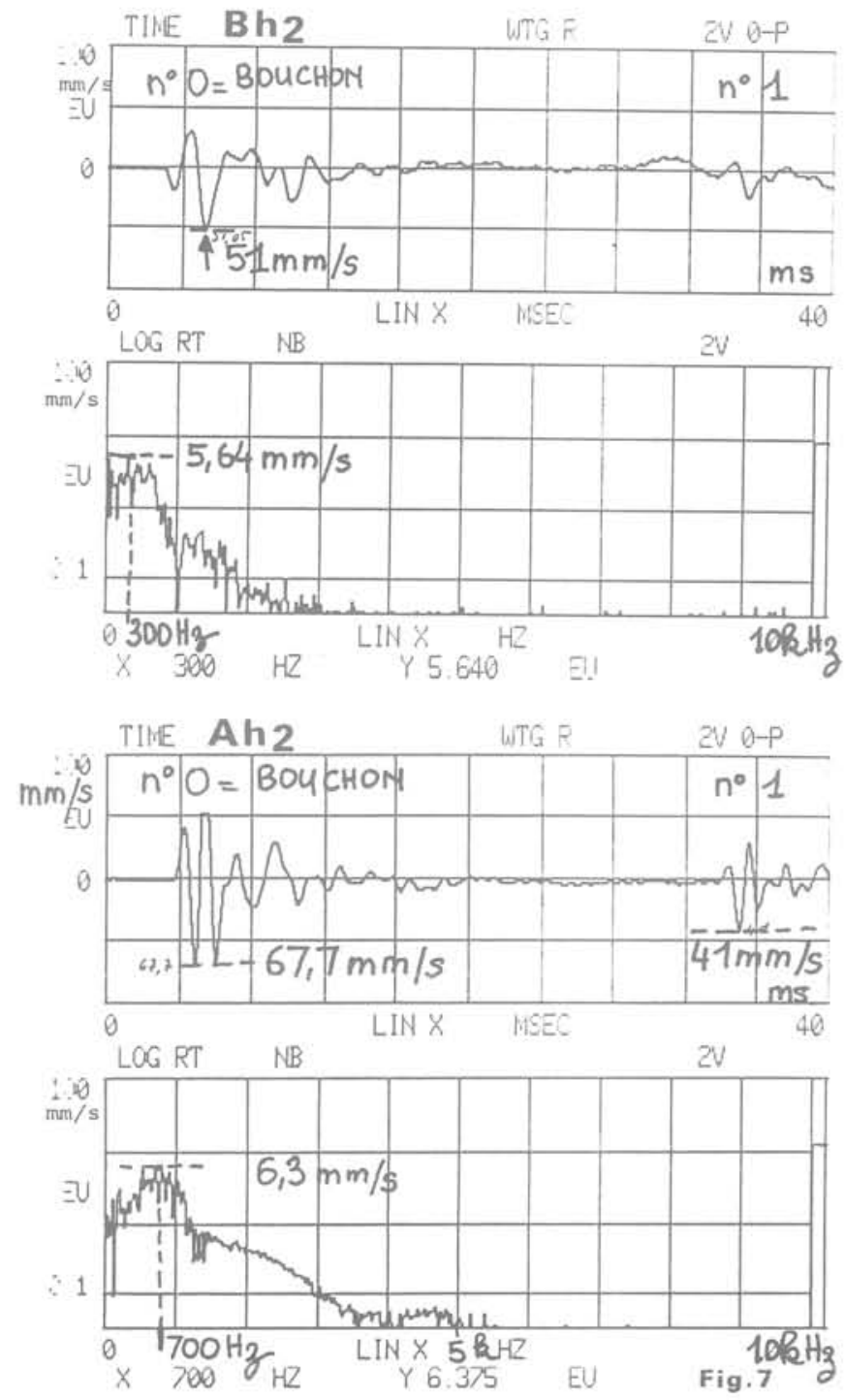

Fig. 7 : Analyse spectrale sur les signaux délivrés par 2 géophones horizontaux perpendiculaires à l'axe du tunnel scellés à une hauteur de $1,5 \mathrm{~m}$ sur les pieds droits et à des distances de $2 \mathrm{~m}$ pour le capteur A et $4 \mathrm{~m}$ pour le capteur B par rapport à l'extrémité de la volée. Celle-ci est constituée par un tir de plot de longrine.

Les signaux présentés correspondent aux micro-retards numéro zéro et $n^{\circ} 1$, qui constituent le bouchon de la volée. Les numéros zéro amorcent 2 forages chargés à $100 \mathrm{~g}$ chacun ; les numéros 1 , amorcent 2 forages chargés à 125 g chacun.

Fig. 8 : Les 2 spectres de fréquences représentent I'analyse sur 100 canaux entre $25 \mathrm{~Hz}$ et $2525 \mathrm{~Hz}$ des 2 signaux représentés sur la fig. 7. Il s'agit en fait d'une dilatation des spectres de cette même figure.

Observations : La plage de fréquences est particulièrement large, en particulier vers les basses fréquences sur la capteur B. Ces niveaux varient peu, sur les canaux 25 à $650 \mathrm{~Hz}$.

Sur le capteur A le plus proche, la fréquence d'amplitude maximale est à $700 \mathrm{~Hz}$, et la décroissance des niveaux vers les basses fréquences est relativement faible.

La fig. 9 représente le rapport entre les 2 spectres précédents. On observera des niveaux sensiblement constants ou très sélectivement amplifiés entre $25 \mathrm{~Hz}$ et $500 \mathrm{~Hz}$, puis une atténuation croissante avec la fréquence entre 500 et $1000 \mathrm{~Hz}$ avec une pente moyenne de $25 \mathrm{~dB}$ par octave.

Au dessus de $1000 \mathrm{~Hz}$ l'atténuation varie de 5 à $20 \mathrm{~dB}$.

Conclusion : Les tirs réalisés en tunnel, tant pour le creusement de niches, que pour l'abaissement de la plate-forme, engendrent sur la section du tunnel des vibrations sur une plage de fréquences très larg

Les fréquences d'amplituđe maximale se situent entre $250 \mathrm{~Hz}$ et $900 \mathrm{~Hz}$.

La plage totale de fréquences dépend très largement de l'orientation de l'axe de mesure. Les fréquences inférieures à $500 \mathrm{~Hz}$ présentent des niveaux relativement elevés sur les pieds droits, suivant la direction perpendiculaire à la paroi $\left(h_{2}\right)$. 

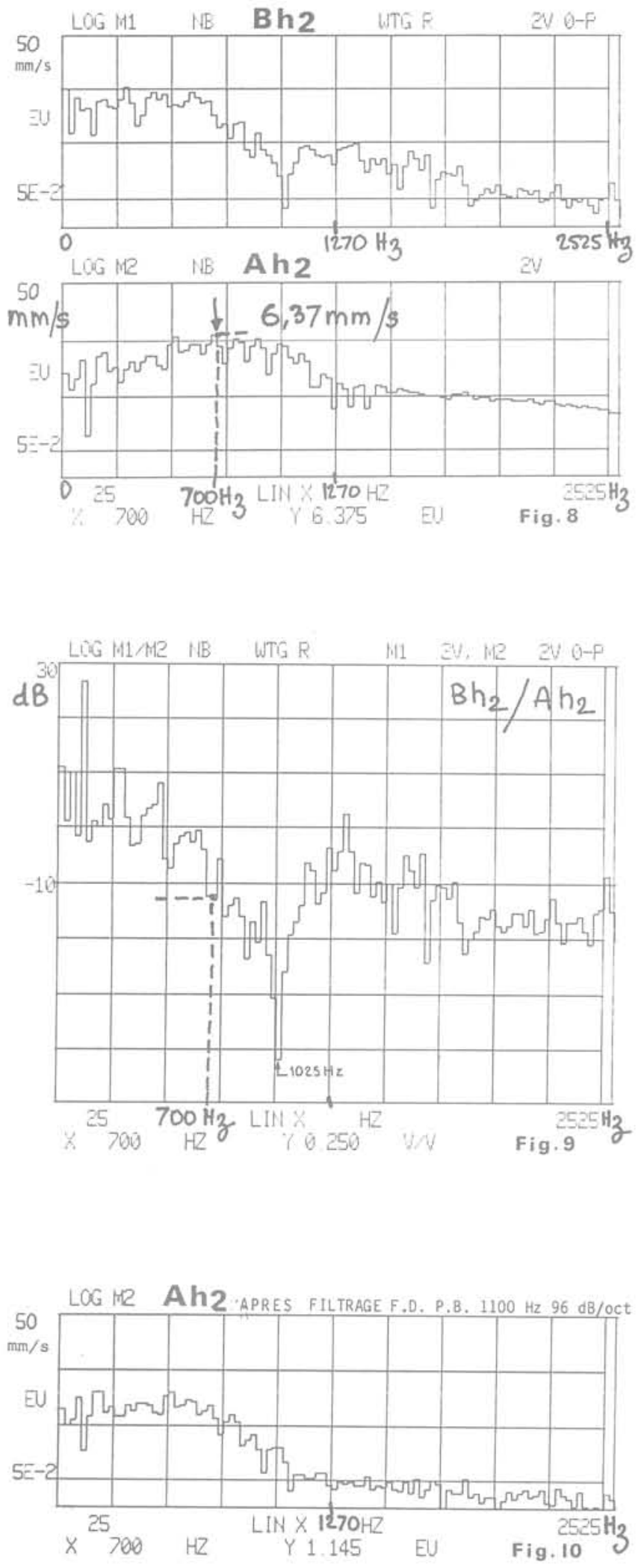

\section{FILTRAGE DES FREQUENCES ELEVEES}

Nous avons vu sur la fig. 9 que, entre les capteurs A et B, nous avions un filtrage des fréquences supérieures à $500 \mathrm{~Hz}$.

Sur les fréquences inférieures à $500 \mathrm{~Hz}$ on observe par contre une sensible amplification.

Appliquons au signal du capteur $\mathrm{A}$, un filtrage passe bas à $1100 \mathrm{~Hz}$, avec une pente d'atténuation de $96 \mathrm{~dB} /$ octave, le spectre alors obtenu sur ce signal filtre est très comparable au spectre du signal B en particulier entre $25 \mathrm{~Hz}$ et $1000 \mathrm{~Hz}$ (fig. 10).

La fig. 11 représente le signal de la première impulsion, en fonction du temps : d'une part sans filtrage, d'autre part avec le filtrage passe-bas à $1100 \mathrm{~Hz}$ (fig. 11) l'atténuation est notable puisque la valeur zéro-crêtes passe de $68 \mathrm{~mm} / \mathrm{s}$ à $10 \mathrm{~mm} / \mathrm{s}$ sur le premier pic négatif.

Sur le signal filtré la valeur zéro crête maximale est obtenue sur le 2 ème pic négatif avec $23,3 \mathrm{~mm} / \mathrm{s}$.

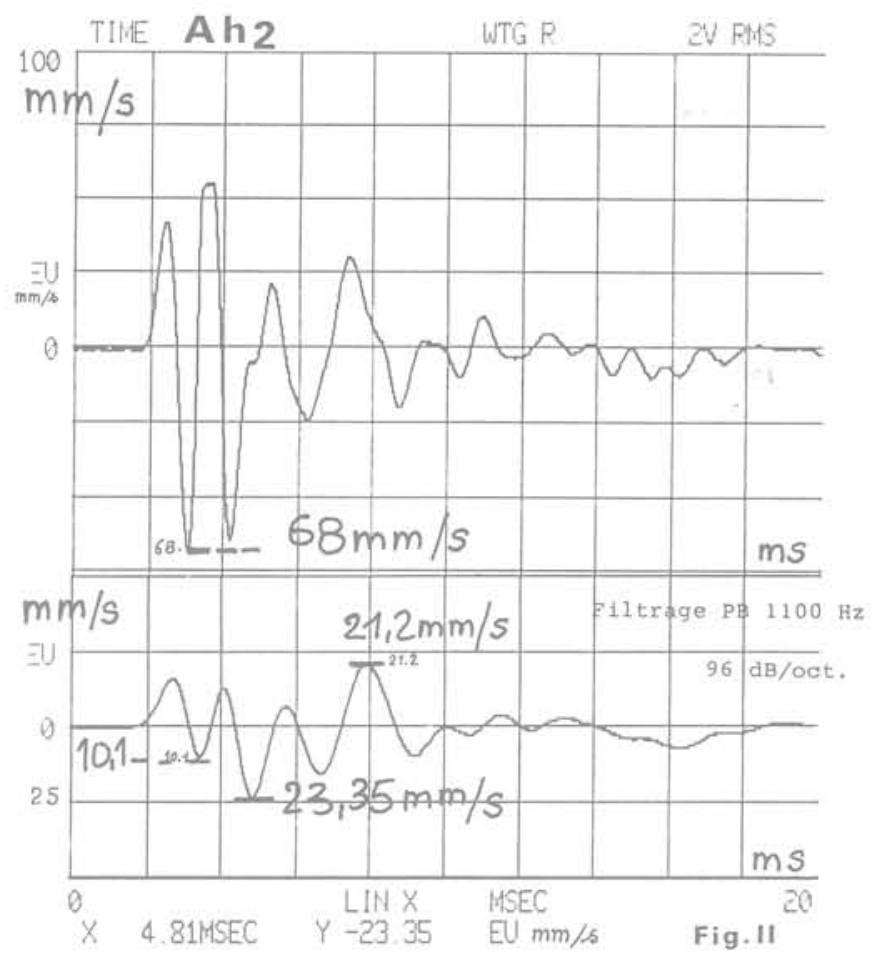

on comprendra dans ces conditions que les mesures de niveaux zéro-crêtes des vitesses de vibrations dans un tunnel sont largement fonction de la bande passante de la chaine de mesure, depuis le capteur et son mode de fixation sur la paroi, jusqu'à la saisie du phénomène. 
La fig. 12 représente 1'ensemble des impulsions enregistrées lors d'un tir de niche, d'une part sur la bande totale d'enregistrement $(0-1250 \mathrm{~Hz})$ puis avec des filtrages passe-bas successivement à $500 \mathrm{~Hz}-300 \mathrm{~Hz}$ $200 \mathrm{~Hz}$ et $100 \mathrm{~Hz}$. Les niveaux zéro-crêtes maxi passent ainsi successivement de $49 \mathrm{~mm} / \mathrm{s}$ à $14 \mathrm{~mm} / \mathrm{s}-3,5 \mathrm{~mm} / \mathrm{s}-1,1 \mathrm{~mm} / \mathrm{s}$ et $0,4 \mathrm{~mm} / \mathrm{s}$.
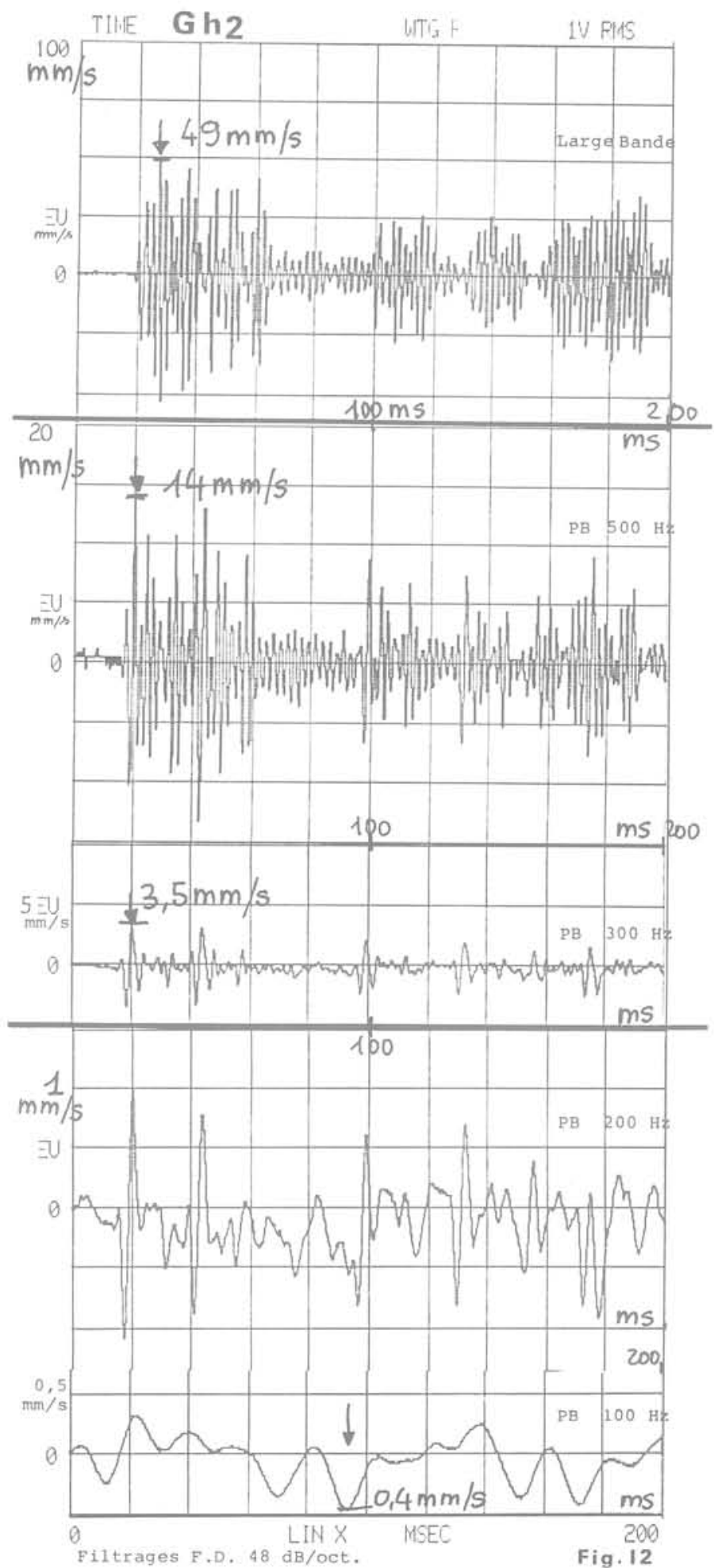

\section{CONCLUSION}

Les tirs de mines réalisés au cours des travaux de reprise en sous oeuvre des tunnels engendrent sur les parements d'une section située entre 0 et 5 mètres de l'extrémité d'une volée, des vibrations sur une plage de fréquences très large entre quelques hertz et $2 \mathrm{KHz}$.

Les fréquences d'amplitude maximale se situent entre 250 et $900 \mathrm{~Hz}$.

Sur la bande de fréquences la plus large, les vibrations atteignent couramment des niveaux élevés de 50 à $100 \mathrm{~mm} / \mathrm{s}$. La détection des vibrations sur une gamme de fréquences supérieures à $500 \mathrm{~Hz}$ et fortement conditionnée par le mode de fixation des capteurs.

Les géophones couramment utilisés ne présentent, pas des caractéristiques toujours très adaptées pour la détection sur des fréquences supérieures à quelques centaines de hertz.

Les caractéristiques des appareils de saisie les plus couramment utilisés ne sont généralement pas connues avec précision dans le domaine des fréquences supérieures à $250 \mathrm{~Hz}$.

Le contrôle et l'efficacité d'un tir, ne peut donc pas être assuré uniquement en déterminant la vitesse de vibration zéro-crête. maximale de la série d'impulsions successives.

Les problèmes de nuisances sont principalement préoccupants sur le domaine des basses fréquences. C'est pourquoi nous préconisons pour les contrôles de tirs de mines en tunnel, une première phase d'ajustage des tirs de mines avec une étude complète des vibrations. Les spectres de fréquences nous permettent d'apprécier le degré de nocivité des vibrations en fonction de la gamme de fréquences. L'amplitude zéro-crête de la vibration, en tant que critère de nocivité, ne pourra être valablement retenue que sur une plage de fréquences, caractérisée par une transmission préférentielle dans l'ouvrage, c'est à dire un amortissement nul ou très lent dans les environs du tir.

L'utilisation de séries souvent complètes de micro-retards, a pour effet d'engendrer sur cette gamme de fréquences généralement basses, une vibration pratiquement entretenue, pendant environ $500 \mathrm{~ms}$.

La connaissance des niveaux efficaces de la vibration pendant le temps de la volée peut également constituer un critère d'appréciation de l'énergie dissipée sous la forme de vibrations dans 1 'ouvrage. 
